\title{
artigo
}

\section{Promoção da saúde de graduandos de enfermagem: uma revisão integrativa}

\author{
Health promotion of nursing graduates: an integrative review \\ Promoción de la salud de graduados deenfermería: una revisión integrativa
}

\begin{abstract}
RESUMO
Objetivo: Analisar as atividades de Promoção da Saúde oferecidas pelas Universidades aos discentes de enfermagem, a partir da produção científica nacional e internacional; e identificar as evidências dessas intervenções tendo em vista o movimento das Universidades Promotoras da Saúde. Métodos: Revisão Integrativa da literatura, com uso dos descritores em saúde, palavras-chave e MeshTerms, realizada nas bases de dados Literatura Latino-Americana e do Caribe em Ciências da Saúde (LILACS), PubMed Central (PMC), Cochrane e Web of Science (WoS), no período de 2003 a 2019. Resultados: Foram selecionados 14 artigos, que foram agrupados nas categorias: Fazer e formar para saúde: avaliação de promoção da saúde e; Outras necessidades de Promoção da Saúde. Conclusões: As estratégias de promoção da saúde oferecidas pelas universidades aos graduandos demonstram preocupação com aspectos pontuais do desenvolvimento do corpo do estudante, mas nem sempre resulta da articulação entre as demandas e o que é oferecido, no que tange a saúde dos universitários. Verificou-se a necessidade de novos estudos sobre a temática, que contribuam para a construção de políticas e ações de saúde voltadas para a população universitária, que necessita ser atendida em sua singularidade.
\end{abstract}

DESCRITORES: Promoção da Saúde; Universidade; Enfermagem; Serviços de Saúde para Estudantes.

\section{ABSTRACT}

Objective: To analyze the Health Promotion activities offered by Universities to nursing students, based on national and international scientific production; and to identify the evidence of these interventions in view of the movement of Health Promoting Universities. Methods: Integrative literature review, using health descriptors, keywords and MeshTerms, carried out in the Latin American and Caribbean Literature databases in Health Sciences (LILACS), PubMed Central (PMC), Cochrane and Web of Science (WoS), from 2003 to 2019. Results: 14 articles were selected, which were grouped into the categories: Doing and training for health: evaluation of health promotion and Other Health Promotion needs. Conclusions: The health promotion strategies offered by universities to undergraduate students show concern with specific aspects of the development of the student's body, but it does not always result from the articulation between the demands and what is offered, regarding the health of university students. There was a need for further studies on the subject, which contribute to the construction of health policies and actions aimed at the university population, which needs to be attended to in its singularity.

DESCRIPTORS: Health Promotion; University; Nursing; Student Health Services.

\section{RESUMEN}

Objetivo: analizar las actividades de promoción de la salud que ofrecen las universidades a los estudiantes de enfermería, con base en la producción científica nacional e internacional; e identificar la evidencia de estas intervenciones en vista del movimiento de las universidades promotoras de la salud. Métodos: Revisión de la literatura integradora, utilizando descriptores de salud, palabras clave y MeshTerms, realizados en las bases de datos de literatura latinoamericana y caribeña en Ciencias de la salud (LILACS), PubMed Central (PMC), Cochrane y Web of Science (WoS), de 2003 a 2019. Resultados: se seleccionaron 14 artículos, que se agruparon en las categorías: Hacer y capacitar para la salud: evaluación de promoción de la salud y; Otras necesidades de promoción de la salud Conclusiones: Las estrategias de promoción de la salud que ofrecen las universidades a los estudiantes de pregrado muestran preocupación por aspectos específicos del desarrollo del cuerpo del estudiante, pero no siempre resulta de la articulación entre las demandas y lo que se ofrece, con respecto a La salud de los estudiantes universitarios. Fue necesario realizar más estudios sobre el tema, que contribuyan a la construcción de políticas y acciones de salud dirigidas a la población universitaria, que deben ser atendidas en su singularidad.

DESCRIPTORES: Promoción de la Salud; Universidad; Enfermería; Servicios de Salud Estudiantil.

RECEBIDO EM: 13/02/2020 APROVADO EM: 14/02/2020 


\section{Beatriz Carvalho Espindola}

Enfermeira. Mestranda em Ciências do Cuidado em Saúde. Escola de Enfermagem Aurora de Afonso Costa. Universidade Federal Fluminense. Niterói, Rio de Janeiro. https://orcid.org/0000-0003-4317-9234

\section{Vera Maria Sabóia}

Enfermeira. Professora titular. Escola de Enfermagem Aurora de Afonso Costa. Universidade Federal Fluminense. Niterói, Rio de Janeiro. https://orcid.org/0000-0003-0382-5078

\section{Gabriela Silva dos Santos}

Doutoranda. Escola de Enfermagem Anna Nery. Universidade Federal do Rio de Janeiro. https://orcid.org/0000-0001-6687-9888

\section{INTRODUÇÃO}

$\mathbf{P}$ or décadas, as universidades vêm desenvolvendo um importante papel na vida de jovens estudantes. Tais instituições são reconhecidas como parte do percurso para advento da construção profissional, que permeia o indivíduo em diferentes fases, momentos e níveis de maturidade e responsabilidade. Assim, torna-se um ambiente privilegiado, agrupando pessoas em uma etapa importante de suas vidas.

A Universidade, como ambiente de formação acadêmica, tem como propósito a formação integral dos profissionais do ponto de vista humanístico, científico e tecnológico, realizando atividades para o desenvolvimento humano sustentável, incluindo estudantes, docentes e técnicos administrativos ${ }^{(1)}$.

A concepção da promoção da saúde alcança uma abrangência muito maior do que a que circunscreve ao campo da saúde, incluindo o ambiente em sentido amplo, ultrapassando a perspectiva local e global, além de incorporar elementos físicos, psicológicos e sociais. Um dos eixos básicos da promoção da saúde é fortalecer a autonomia dos sujeitos e dos grupos sociais, pois ensinar não é transferir um conhecimento absoluto, mas sim possibilitar que essa construção seja realizada pelo estudante universitário ${ }^{(2)}$.

A Organização Mundial de Saúde (OMS), no ano de 1998, publicou um guia de orientação para universidades que objetivava promoção da saúde, explicitando seus contextos e contribuições. Em 2003 , teve início a formalidade institucional do movimento das Universidades Promotoras de Saúde (UPS) na América Latina, com alcance Iberoamericano. Trata-se de uma iniciativa adequada para fortalecer a saúde mediante ao ideal e as práticas da promoção da saúde ${ }^{(3)}$.

As Universidades Promotoras de Saúde (UPS) são espaços privilegiados para a criação de um contexto promotor de saúde para a comunidade educativa, pois melhoram o perfil de saúde por meio do desenvolvimento do ensino, da investigação e do compartilhamento de conhecimentos, o que contribui, também, para o bem-estar e sustentabilidade da comunidade em geral e, ainda, para a avaliação da eficácia dos programas de intervenção ${ }^{(4)}$.

Ao longo da história, importantes documentos foram elaborados a partir de conferências e congressos, a fim de melhorar a realidade em saúde dos estudantes universitários. Dentre os mais recentes, podemos destacar a Carta de Okanagan, que estimula a incorporação dos princípios de saúde e bem-estar nas atividades cotidianas realizadas nas universidades promotoras da saúde e a Declaração de Alicante, que por sua vez, destaca a participação das universidades em decisões que afetam a saúde, incluindo o ambiente no qual os seus participantes trabalham, vivam e se relacionam ${ }^{(4,5)}$.

A melhor educação é aquela capaz de promover a participação dos sujeitos, incentivando-os a reflexão, o diálogo, a criatividade e sua autonomia, empoderando-os durante todo o processo de ensino-aprendizagem. Não se trata de um favor que pode ou não ser concedido, não considerar a curiosidade, preferências, inquietudes e linguagens do educando é ir contra os princípios éticos da educação e reprime sua liberdade ${ }^{(6)}$.

A saúde do educando é criada e vivida por pessoas dentro das configurações de sua vida cotidiana; onde elas aprendem, trabalham, brincam e amam ${ }^{(4)}$. Configurações Saudáveis é um conceito que engloba abordagens baseadas em configurações de Promoção da Saúde, envolve um método holístico e multidisciplinar que integra a ação entre os fatores de risco. As abordagens de Configuração Saudável são implementadas de diferentes maneiras e em vários ambientes tais como escolas, universidades, locais de trabalho e hospitais ${ }^{(7)}$.

$\mathrm{Na} 9^{a}$ Conferência Global sobre Promoção da Saúde, líderes globais concordaram em promover a saúde visando alcançar o Desenvolvimento Sustentável. A Conferência destacou a necessidade de ação política governamental que proteja a população dos riscos à saúde, forneça acesso a escolhas saudáveis e divulgue a consciência de como ser e se manter saudável( ${ }^{(8)}$.

Nesse sentido de implementação do movimento das Universidades Promotoras da Saúde, seria desejável que as instituições concebessem e implementassem estratégias e ações que envolvessem os estudantes, docentes, servidores e os recursos locais para garantir sua sustentabilidade ${ }^{(9)}$.

A experiência educativa, a capacidade de aprender deve ir além da simples adaptação à realidade imposta ${ }^{(6)}$. Após estes levantamentos, o objetivo definido para o estudo foi: analisar as atividades de Promoção da Saúde oferecidas pelas Universidades aos discentes de enfermagem, a partir da produção científica nacional e internacional; e identificar as evidências dessas intervenções tendo em vista o movimento das Universidades Promotoras da Saúde.

A questão que norteou o estudo foi: Quais são as evidências científicas sobre as práticas de Promoção da Saúde oferecidas pelas Universidades aos discentes de enfermagem? 


\section{artigo}

\section{METODOLOGIA}

A fim de alcançar os objetivos propostos, optou-se por um estudo de revisão integrativa que consiste em ampla análise de publicações, com a finalidade de obter dados sobre determinada temática e gerar um conhecimento. Esse tipo de pesquisa inclui a análise de publicações relevantes, possibilita a síntese de estudos publicados sobre o assunto, indica lacunas do conhecimento que precisam ser preenchidas por meio de novas pesquisas, além de proporcionar conclusões gerais a respeito da área do estudo ${ }^{(10)}$.

A revisão integrativa é dividida em seis etapas, sendo elas: 1) identificação do tema e seleção da questão de pesquisa; 2) estabelecimento dos critérios de inclusão e exclusão; 3)identificação dos estudos pré-selecionados e selecionados; 4) categorização dos estudos selecionados; 5) análise e interpretação dos resultados; e 6) apresentação da revisão/síntese do conhecimento ${ }^{(11)}$.

A seleção dos estudos foi realizada pelos autores em janeiro de 2020 por meio do acesso online de artigos, indexados nas bases de dados Biblioteca Virtual em Saúde (BVS), PubMed Central (PMC), Cochrane e Web of Science (WoS).
Buscou-se contemplar a compreensão da temática, optando pelo cruzamento dos descritores indexados no sistema de Descritores em Ciência da Saúde - DeCS (promoção da saúde, universidades, enfermagem e serviços de saúde para estudantes) e no Medical SubjectHeadings - MeSH (health promotion, promotion of health, universities, university, nursing, student health services e student health service). Para a realização da pesquisa, utilizaram-se também os Operadores Booleanos "AND" e "OR".

O processo de seleção de artigos respeitou como critérios de inclusão artigos publicados nos últimos 17 anos, nos idiomas português, inglês e espanhol, diretamente relacionados à temática e artigos com resultados de pesquisa respondendo à questão norteadora do estudo em tela. Quanto ao corte temporal, optou-se por buscar publicações de Janeiro de 2003 a Dezembro de 2019, uma vez que em 2003 teve o início da formalidade institucional do movimento das UPS, com participação de ações de promoção da saúde, na América Latina e alcance Iberoamericano, em países como Chile, Colômbia, Costa Rica, Cuba, México e Peru ${ }^{(3)}$.
Os critérios de exclusão definidos foram artigos repetidos em mais de uma base de dados, artigos com atividades de promoção da saúde que não contemplassem os discentes de enfermagem ou comportamentos arriscados desses discentes, sem que houvesse atividades de promoção da saúde relacionadas a esses agravos e estudos que não tivessem conformidade com o objetivo do estudo.

Inicialmente foram selecionados 338 artigos, após a inserção dos critérios de inclusão este número reduziu para 251 artigos. Em seguida, foi realizada a leitura dos títulos e resumos e excluídos 192 artigos por falta de conformidade com o objetivo do estudo. Dentre estes artigos excluídos pode-se dividir o motivo de exclusão em 2 categorias principais: artigos que estavam relacionados a práticas e atividades desenvolvidas por graduandos de enfermagem, com o objetivo de promoção da saúde da comunidade e atividades desenvolvidos em instituições escolares.

Procedeu-se a leitura de cada artigo na íntegra, sendo excluídas 41 publicações que não respondiam ao questionamento $\mathrm{e}$ ao objetivo do estudo. Tais artigos focavam nas experiências de estudantes e enfermei-

\section{Quadro 1. Quadro com as estratégias de busca nas bases de dados utilizados neste estudo. Niterói, RJ, Brasil, 2020.}

\begin{tabular}{|c|c|}
\hline $\begin{array}{l}\text { BASE DE } \\
\text { DADOS }\end{array}$ & ESTRATÉGIA DE BUSCA \\
\hline BVS & $\begin{array}{l}\text { (tw:(promoção da saúde)) AND (tw:(universidades)) AND (tw:(enfermagem)) AND (tw:(serviços de saúde para estudan- } \\
\text { tes)) AND ( la:("en" OR "pt" OR "es")) AND (year_cluster:[2003 TO 2019]) }\end{array}$ \\
\hline PubMed & $\begin{array}{l}\text { (/("health promotion"[MeSH Terms] OR ("health"[All Fields] AND "promotion"[All Fields]) OR "health promotion"[All } \\
\text { Fields]) OR ("health promotion"[MeSH Terms] OR ("health"[All Fields] AND "promotion"[All Fields]) OR "health } \\
\text { promotion"[All Fields] OR ("promotion"[All Fields] AND "health"[All Fields]) OR "promotion of health"[All Fields])) AND } \\
\text { (("universities"[MeSH Terms] OR "universities"[All Fields]) OR ("universities"[MeSH Terms] OR "universities"[All Fields] } \\
\text { OR "university"[All Fields])) AND ("nursing"[Subheading] OR "nursing"[All Fields] OR "nursing"[MeSH Terms] OR } \\
\text { "nursing"[All Fields] OR "breast feeding"[MeSH Terms] OR ("breast"[All Fields] AND "feeding"[All Fields]) OR "breast } \\
\text { feeding"[All Fields])) AND (("student health services"[MeSH Terms] OR ("student"[All Fields] AND "health"[All Fields] } \\
\text { AND "services"[All Fields]) OR "student health services"[All Fields]) OR ("student health services"[MeSH Terms] OR } \\
\text { ("student"[All Fields] AND "health"[All Fields] AND "services"[All Fields]) OR "student health services"[All Fields] OR } \\
\text { ("student"[All Fields] AND "health"[All Fields] AND "service"[All Fields]) OR "student health service"[All Fields])) AND } \\
\text { (("2003/01/01"[PDAT] : "2019/12/31"[PDAT]) AND (Spanish[lang] OR Portuguese[lang] OR English[lang])) }\end{array}$ \\
\hline Cochrane & $\begin{array}{l}\text { health promotion in Title Abstract Keyword AND universities in Title Abstract Keyword AND nursing in Title Abstract } \\
\text { Keyword AND student health services in Title Abstract Keyword - with Cochrane Library publication date Between Jan } \\
2003 \text { and Dec } 2019 \text { (Word variations have been searched) }\end{array}$ \\
\hline WoS & $\begin{array}{l}\text { TÓPICO: (health promotion) AND TÓPICO: (universities) AND TÓPICO: (nursing) AND TÓPICO: (student health services) } \\
\text { Refinado por: ANOS DE PUBLICAÇÃO: (2014 OR } 2008 \text { OR } 2019 \text { OR } 2013 \text { OR } 2007 \text { OR } 2018 \text { OR } 2012 \text { OR } 2006 \text { OR } 2017 \\
\text { OR } 2010 \text { OR } 2004 \text { OR } 2016 \text { OR 2009 OR 2015) AND IDIOMAS: (ENGLISH OR PORTUGUESE OR SPANISH) }\end{array}$ \\
\hline
\end{tabular}


Figura 1. Fluxograma de identificação, seleção e inclusão dos estudos da revisão integrativa. Niterói, RJ, Brasil, 2020.

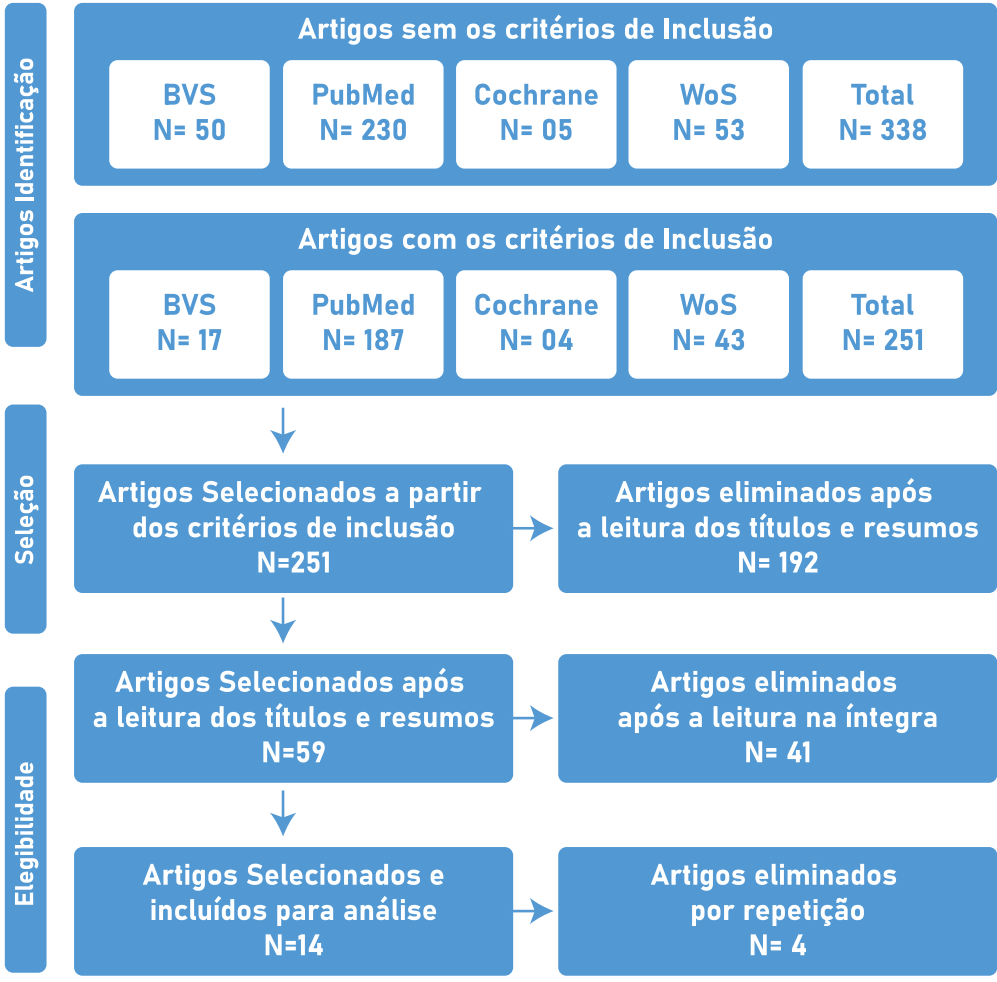

ros no campo prático de ensino, ou era voltado para promoção da saúde de estudantes escolares ou avaliaram comportamentos arriscados dos discentes universitários, sem que houvesse nenhuma atividade na universidade para minimizar tais comportamentos. 04 artigos estavam duplicados em mais de uma Base de Dados.

Sendo assim, o corpus de análise ficou caracterizado em 14 artigos científicos que discorrem sobre a Promoção da Saúde com universitários de enfermagem. $\mathrm{O}$ fluxograma de identificação apresenta o processo de seleção dos estudos (Figura 1).

\section{RESULTADOS}

Entre as produções encontradas, foram selecionados 14 artigos que contemplavam aos critérios de inclusão e exclusão, baseados na temática "Promoção da Saúde oferecidas pelas Universidades aos discentes de enfermagem". Esses artigos são apresentados no Quadro 2, no qual se descrevem as seguintes características da publicação (autores, bases de dados, país, título, objetivo e conclusão).

Quadro 2. Caracterização dos artigos segundo autores, bases de dados, país, título, objetivo e conclusão. Niterói, RJ, Brasil, 2020

\begin{tabular}{|c|c|c|c|c|}
\hline $\begin{array}{l}\text { AUTORES/ BASE } \\
\text { DE DADOS/ ANO }\end{array}$ & PAís & TÍTULO & OBJETIVOS & CONCLUSÃO \\
\hline $\begin{array}{l}\text { Aparicio CXP, } \\
\text { Hernandez FJB, } \\
\text { Tamayo YG }{ }^{(12)} \\
\text { WOS } \\
2019\end{array}$ & Equador & $\begin{array}{l}\text { Modelo de promoção } \\
\text { da saúde na Universi- } \\
\text { dade Metropolitana do } \\
\text { Equador }\end{array}$ & $\begin{array}{l}\text { Apresentar um modelo } \\
\text { de promoção da saúde } \\
\text { a ser aplicado na Uni- } \\
\text { versidade Metropolita- } \\
\text { na do Equador. }\end{array}$ & $\begin{array}{l}\text { O modelo apresentado constitui uma estraté- } \\
\text { gia para aprofundar a maneira como os indi- } \\
\text { víduos tomam decisões sobre seus cuidados } \\
\text { de saúde. As ações realizadas pelo grupo de } \\
\text { apoio especializado demonstram a busca para } \\
\text { se atingir os princípios de promoção da saúde } \\
\text { nesse centro de ensino superior. }\end{array}$ \\
\hline $\begin{array}{l}\text { Burgess AW, } \\
\text { Thabault P, Kiernan } \\
\text { L, Flaherty } E_{1} \\
\text { Leestma K(13). } \\
\text { PUBMED } \\
2019\end{array}$ & EUA & $\begin{array}{l}\text { Iniciativa Atleta } \\
\text { Guerreiro Universitário } \\
\text { e a Enfermagem } \\
\text { Acadêmica }\end{array}$ & $\begin{array}{l}\text { Determinar se um } \\
\text { elemento-chave da } \\
\text { cultura ocupacional } \\
\text { militar é capaz de } \\
\text { preparar atletas } \\
\text { universitários com } \\
\text { veteranos }\end{array}$ & $\begin{array}{l}\text { Este programa de intervenção no exercício } \\
\text { físico foi implementado em dois Campus } \\
\text { Universitários e indica os benefícios de } \\
\text { envolver Membros Veteranos do Serviço } \\
\text { Militar com o Serviço de Promoção da Saúde } \\
\text { dos estudantes Universitários para melhoria } \\
\text { da saúde. }\end{array}$ \\
\hline $\begin{array}{l}\text { Liang HF, Wu KM, } \\
\text { Hung CC, Wang YH, } \\
\text { Peng NH }{ }^{(14)} \text {. } \\
\text { PUBMED } \\
2019\end{array}$ & Taiwan & $\begin{array}{l}\text { Aumento da resiliência } \\
\text { entre estudantes de } \\
\text { enfermagem durante } \\
\text { as práticas clínicas: um } \\
\text { estudo participativo de } \\
\text { pesquisa-ação. }\end{array}$ & $\begin{array}{l}\text { Desenvolver e imple- } \\
\text { mentar um projeto base- } \\
\text { ado em aprimoramento } \\
\text { da resiliência (ER) para } \\
\text { estudantes de enferma- } \\
\text { gem de Taiwan durante o } \\
\text { estágio em Last Mile. }\end{array}$ & $\begin{array}{l}\text { O projeto de ER ajudou os alunos a desen- } \\
\text { volver resiliência, aprimorando seus conhe- } \\
\text { cimentos e habilidades de enfermagem e } \\
\text { praticando pensamentos e comportamentos } \\
\text { positivos. }\end{array}$ \\
\hline
\end{tabular}




\section{artigo}

\begin{tabular}{|c|c|c|c|c|}
\hline $\begin{array}{l}\text { Wichmann F, } \\
\text { Braun M, Ganz T, } \\
\text { Lubasch J, Heiden- } \\
\text { reich T, Laging M, } \\
\text { Pischke CR }{ }^{(15)} \text {. } \\
\text { PUBMED } \\
2019\end{array}$ & Alemanha & $\begin{array}{l}\text { Avaliação no Campus da } \\
\text { prontidão da comunida- } \\
\text { de na implementação } \\
\text { de programas on-line } \\
\text { baseados em evidên- } \\
\text { cias para evitar o uso de } \\
\text { substâncias de risco en- } \\
\text { tre os estudantes uni- } \\
\text { versitários na Alemanha }\end{array}$ & $\begin{array}{l}\text { Avaliar o nível de pron- } \\
\text { tidão (capacidade) de } \\
\text { universidades alemãs } \\
\text { relativas à execução dos } \\
\text { programas on-line ba- } \\
\text { seadas em evidências } \\
\text { para a prevenção do uso } \\
\text { de substâncias de risco }\end{array}$ & $\begin{array}{l}\text { A avaliação é bem adequada para contribuir } \\
\text { para os atuais desafios de integração de pro- } \\
\text { gramas on-line baseados em evidências, que } \\
\text { têm o potencial de atingir grupos de difícil al- } \\
\text { cance (por exemplo, alunos fora do campus) } \\
\text { em atividades de promoção da saúde existen- } \\
\text { tes nas universidades. }\end{array}$ \\
\hline $\begin{array}{l}\text { McCabe } \mathrm{B} \text {, Troy } \mathrm{A}, \\
\text { Patel } \mathrm{H}, \mathrm{Hals} \text { stead } \mathrm{V}, \\
\text { Arana } \mathrm{M}^{(16)} \text {. } \\
\text { PUBMED } \\
2018\end{array}$ & EUA & $\begin{array}{l}\text { Desenvolvimento } \\
\text { de um programa de } \\
\text { promoção do sono } \\
\text { saudável no campus } \\
\text { universitário }\end{array}$ & $\begin{array}{l}\text { Expandir o pequeno } \\
\text { corpo existente } \\
\text { da literatura sobre } \\
\text { intervenções do sono } \\
\text { para estudantes } \\
\text { universitários }\end{array}$ & $\begin{array}{l}\text { Houve melhora na percepção da importância } \\
\text { do sono dos alunos de graduação e a qualidade } \\
\text { do sono melhorou significativamente após o } \\
\text { workshop. }\end{array}$ \\
\hline $\begin{array}{l}\text { Sawicki WC, } \\
\text { Fram DS, } \\
\text { Belasco AGS(17). } \\
\text { BVS/ } \\
\text { LILACS } \\
2018\end{array}$ & Brasil & $\begin{array}{l}\text { Intervenção Breve apli- } \\
\text { cada a universitários } \\
\text { consumidores de bebi- } \\
\text { das alcoólicas }\end{array}$ & $\begin{array}{l}\text { Investigar o consumo } \\
\text { de álcool entre universi- } \\
\text { tários de enfermagem e } \\
\text { avaliar intervenção bre- } \\
\text { ve para abusadores de } \\
\text { álcool. }\end{array}$ & $\begin{array}{l}\text { A Intervenção Breve diminui significativamen- } \\
\text { te o padrão de consumo de álcool, promoven- } \\
\text { do a saúde. Medidas de prevenção do uso e } \\
\text { abuso de álcool devem ser programadas, em } \\
\text { especial, visando à prevenção de problemas } \\
\text { decorrentes do consumo excessivo do álcool } \\
\text { e promoção da saúde física e psicossocial. }\end{array}$ \\
\hline $\begin{array}{l}\text { Cook } L^{(18)} \\
\text { BVS/ } \\
\text { PUBMED } \\
2011\end{array}$ & EUA & $\begin{array}{l}\text { O papel da enfermeira } \\
\text { psiquiátrica na criação } \\
\text { de um programa de pre- } \\
\text { venção do suicídio no } \\
\text { campus }\end{array}$ & $\begin{array}{l}\text { Descrever como um } \\
\text { membro da faculdade } \\
\text { de enfermagem psiqui- } \\
\text { átrica estabeleceu com } \\
\text { sucesso um programa } \\
\text { abrangente de preven- } \\
\text { ção ao suicídio. }\end{array}$ & $\begin{array}{l}\text { Atualmente o suicídio é identificado como a se- } \\
\text { gunda principal causa de morte em estudantes } \\
\text { universitários. Muitos campi universitários fo- } \\
\text { ram capazes de estabelecer programas de pre- } \\
\text { venção do suicídio. }\end{array}$ \\
\hline $\begin{array}{l}\text { Manderscheid RW, } \\
\text { Masi D, Rossignol } \\
\text { CR, MasiDA }{ }^{(19)} \text {. } \\
\text { BVS/ } \\
\text { PUBMED } \\
2007\end{array}$ & EUA & $\begin{array}{l}\text { A integração dos servi- } \\
\text { ços de saúde física e de } \\
\text { saúde comportamental: } \\
\text { três exemplos de casos } \\
\text { universitários }\end{array}$ & $\begin{array}{l}\text { Identificar as caracte- } \\
\text { rísticas dos três estu- } \\
\text { dos de casos sobre a in- } \\
\text { tegração da saúde física } \\
\text { e os serviços de saúde } \\
\text { comportamental. }\end{array}$ & $\begin{array}{l}\text { O estudo mostra não só que a visão de um cuida- } \\
\text { do primário integrado de saúde comportamental } \\
\text { é possível dentro de uma universidade de saúde, } \\
\text { mas também que pode ser feito para fins de me- } \\
\text { Ihora da clínica dos estudantes e dos gastos. }\end{array}$ \\
\hline $\begin{array}{l}\text { Sanders LB }{ }^{(20)} \text {. } \\
\text { PUBMED } \\
2007\end{array}$ & EUA & $\begin{array}{l}\text { Prestação de serviços } \\
\text { de Saúde Mental no } \\
\text { Campus de Long Island: } \\
\text { um estudo piloto. }\end{array}$ & $\begin{array}{l}\text { Descrever a prestação } \\
\text { de serviços de saúde } \\
\text { mental a estudantes que } \\
\text { frequentam faculdades } \\
\text { em Long Island }\end{array}$ & $\begin{array}{l}\text { Estudantes universitários, frequentemente fora de } \\
\text { casa pela primeira vez, correm o risco de distúrbios } \\
\text { de saúde mental que podem afetar o desempenho } \\
\text { acadêmico e a qualidade de vida. Os enfermeiros } \\
\text { empregados nos centros de saúde das faculdades } \\
\text { podem trabalhar com estudantes e familias no } \\
\text { sentido de promover comportamentos de saúde. }\end{array}$ \\
\hline $\begin{array}{l}\text { Sturrock CJ, Currie } \\
\text { MJ, Vally H, O'keefe } \\
\text { EJ, Primrose R, Ha- } \\
\text { bel P, Schamburg } \\
\text { K, Bowden F F(21). } \\
\text { WOS } \\
2007\end{array}$ & Austrália & $\begin{array}{l}\text { Trabalhos de saúde se- } \\
\text { xual na comunidade: } \\
\text { uma revisão do Progra- } \\
\text { ma de Extensão }\end{array}$ & $\begin{array}{l}\text { Oferecer assistência à } \\
\text { saúde sexual em vários } \\
\text { locais de circulação dos } \\
\text { grupos envolvidos }\end{array}$ & $\begin{array}{l}\text { As colaborações entre as agências para fornecer } \\
\text { serviços de extensão facilitam a educação em } \\
\text { saúde sexual na comunidade e a triagem para } \\
\text { grupos com maior risco de IST. Os resultados } \\
\text { também destacam a necessidade de continuar } \\
\text { a vacinação contra hepatite, testes, promoção } \\
\text { da saúde e educação nessas populações. }\end{array}$ \\
\hline
\end{tabular}




\begin{tabular}{|c|c|c|c|c|}
\hline $\begin{array}{l}\text { Canales MK, Carr- } \\
\text { J,Wohlberg, J(22). } \\
\text { PUBMED } \\
2005\end{array}$ & EUA & $\begin{array}{l}\text { "Chegou a hora": per- } \\
\text { cepções das mulheres } \\
\text { universitárias sobre } \\
\text { saúde ginecológica }\end{array}$ & $\begin{array}{l}\text { Examinar as percepções } \\
\text { das mulheres universi- } \\
\text { tárias quanto à saúde } \\
\text { ginecológica, com ênfa- } \\
\text { se particular na tomada } \\
\text { de decisão em saúde. }\end{array}$ & $\begin{array}{l}\text { O período da faculdade pode ser "o momento" } \\
\text { em que as universitárias começam a pensar } \\
\text { e a tomar decisões sobre a saúde ginecológi- } \\
\text { ca, reconhecendo as definições sobre saúde e } \\
\text { comportamentos saudáveis. As enfermeiras } \\
\text { podem ajudar as estudantes a desenvolver } \\
\text { estratégias para implementar essas defini- } \\
\text { ções e integrar os comportamentos saudáveis } \\
\text { desejados em suas vidas universitárias. }\end{array}$ \\
\hline $\begin{array}{l}\text { Geary DM,Nardi } \\
\text { DA, Smith J, } \\
\text { Kremer M }{ }^{(23)} \text {. } \\
\text { PUBMED } \\
2005\end{array}$ & EUA & $\begin{array}{l}\text { Necessidades de } \\
\text { serviços de saúde } \\
\text { mental baseado } \\
\text { na comunidade } \\
\text { universitária: } \\
\text { identificando as } 5 \\
\text { principais }\end{array}$ & $\begin{array}{l}\text { Determinar as } \\
\text { necessidades de } \\
\text { serviços de saúde } \\
\text { mental e de promoção } \\
\text { da saúde prestados pelo } \\
\text { corpo docente avançado } \\
\text { de enfermagem }\end{array}$ & $\begin{array}{l}\text { Indicou-se uma forte necessidade por serviços } \\
\text { de saúde mental (particularmente para } \\
\text { depressão), promoção da saúde, informações } \\
\text { de bem-estar e cuidados primários de } \\
\text { saúde estavam entre as principais áreas de } \\
\text { necessidade entre os discentes de enfermagem. }\end{array}$ \\
\hline $\begin{array}{l}\text { Clemmens D, En- } \\
\text { gler A, Chinn PL }{ }^{(24)} \text {. } \\
\text { BVS/ PUBMED/ } \\
2004\end{array}$ & EUA & $\begin{array}{l}\text { Aprender e viver a saú- } \\
\text { de: experiências de es- } \\
\text { tudantes universitários } \\
\text { com um curso de saúde } \\
\text { introdutório }\end{array}$ & $\begin{array}{l}\text { Avaliar em que medida a } \\
\text { participação no curso de } \\
\text { "Introdução à Saúde" e } \\
\text { atividades relacionadas } \\
\text { influenciou os compor- } \\
\text { tamentos relacionados } \\
\text { à saúde dos alunos. }\end{array}$ & $\begin{array}{l}\text { O campus da faculdade fornece um micro- } \\
\text { cosmo dos problemas de saúde que a nação } \\
\text { enfrenta, com relatos sobre comportamen- } \\
\text { tos de risco, evidentes em taxas mais altas } \\
\text { de fumo e consumo excessivo de álcool. Os } \\
\text { docentes das escolas de enfermagem podem } \\
\text { desenvolver e fornecer programas de promo- } \\
\text { ção da saúde e prevenção de doenças, tanto } \\
\text { para seus próprios alunos enfermeiros, quan- } \\
\text { to para uma população estudantil mais ampla } \\
\text { em todo o campus. }\end{array}$ \\
\hline $\begin{array}{l}\text { Gosline MB, } \\
\text { Schank MJ(25). } \\
\text { BVS/ PUBMED/ } \\
2003\end{array}$ & EUA & $\begin{array}{l}\text { Uma feira de saúde em } \\
\text { toda universidade: um } \\
\text { estágio clínico de pro- } \\
\text { moção da saúde }\end{array}$ & $\begin{array}{l}\text { O objetivo geral foi me- } \\
\text { Ihorar nos participantes } \\
\text { as práticas de autocui- } \\
\text { dado para promoção da } \\
\text { saúde. }\end{array}$ & $\begin{array}{l}\text { A feira de saúde forneceu exibições e recursos } \\
\text { educacionais, foi uma abordagem inovadora } \\
\text { para promoção da saúde e prevenção das doen- } \\
\text { ças na comunidade universitária. }\end{array}$ \\
\hline
\end{tabular}

Após a leitura dos 14 artigos na íntegra, realizou-se uma análise para extração de conceitos abordados em cada artigo e voltados para a temática, possibilitando a elaboração $4^{a}$ etapa da Revisão Integrativa, ou seja, a categorização. Conforme cada categoria levantada, deu-se continuidade a $5^{a}$ etapa da Revisão, análise e interpretação ${ }^{(11)}$.

Os artigos foram agrupados por similaridade do conteúdo e as atividades envolvem o desenvolvimento de programas, cursos, consultas e encontros. A partir da leitura do corpus dos artigos e análise de seus resultados e discussões foram construídas duas categorias de análise: "Fazer e formar para saúde" e "Outras demandas de Promoção da Saúde”.

Na primeira categoria, "Fazer e formar para a saúde: avaliação de promoção da saúde", encontram-se artigos relacionados à implementação, descrição ou avaliação de determinados programas desenvolvidos nestas instituições, voltados para a promoção da saúde dos graduandos de enfermagem.

A implementação de um programa de exercícios físicos nos Estados Unidos da América (EUA) integrou Veteranos de Guerra com os Universitários, de forma a beneficiar a saúde física e mental de ambos os grupos. Os incentivos para a participação no programa e a prática dos exercícios incluíam a oportunidade dos graduandos de enfermagem utilizar as instalações de exercícios do campus, bem como utensílios para uso durante e após as atividades, exemplo, garrafas de água, equipamentos esportivos, vestuário para a prática (camisas e shorts) e cadernos ${ }^{(13)}$.

A implementação de um programa universitário aprimorou a Resiliência dos estudantes de enfermagem. $\mathrm{O}$ projeto de Resiliência teve seis workshops realizados ao longo de dois meses. Os métodos de avaliação envolveram discussão em grupo, entrevistas individuais e diários reflexivos ${ }^{(14)}$.

O ensino teórico-prático é um componente essencial da preparação dos estudantes de enfermagem para aplicar o conhecimento teórico em um ambiente da vida real. No entanto, os alunos geralmente atribuem a esta prática como extremamente estressante, o que pode prejudicar seu desempenho clínico, afetar a qualidade do atendimento ao paciente que presta, bem como sua própria saúde física e psicológica , e dificultar sua continuação nas carreiras de enfermagem. A resiliência, entendida como a capacidade de superar desafios, é fundamental para esses alunos ${ }^{(14)}$ 
Um programa on-line foi implementado com potencial para atingir grupos de difícil alcance para Promoção da Saúde dos universitários, incluindo os de enfermagem. No geral, as universidades incluídas neste estudo exibiam baixos níveis de prontidão para implementação de programas on-line para a prevenção e/ou redução do uso de substâncias arriscadas. Com base nos resultados da avaliação, as universidades receberam feedback sobre as próximas etapas do programa, com vistas para aumentar o envolvimento da instituiçãa ${ }^{(15)}$

Foi avaliado um programa de saúde do sono no campus para estudantes universitários. Nesse estudo, foram realizados 5 grupos focais e 4 oficinas. Os resultados apontaram que mais da metade dos estudantes (59\%) relataram dormir 5-6h por noite, $24 \%$ dormiam sete ou mais horas e $18 \%$ relataram dormir quatro ou menos horas. As oficinas contaram com um facilitador que descrevia a relação entre sono e os acadêmicos, demonstrando como realizar o relaxamento, a meditação e a ioga para aumentar a qualidade do sono ${ }^{(16)}$

Outro estudo apresenta estratégias promotoras de estilos de vida saudáveis e uma preocupação crescente com as taxas de sobrepeso, depressão, diabetes, hipertensão, homicídio e suicídio. $\mathrm{O}$ artigo descreve uma pesquisa conduzida pelo corpo docente durante o primeiro ano de implementação de um programa projetado para avaliar em que medida a participação em atividades na universidade influenciavam comportamentos relacionados à saúde ${ }^{(24)}$. É sugerido que o currículo apoie estilos de vida saudáveis dos estudantes de enfermagem.

Um dos estudos discorre sobre um programa realizado na universidade sobre prevenção ao suicídio, agravo identificado como a segunda principal causa de morte entre os estudantes universitários. O programa que previne o suicídio no campus universitário é desenvolvido pelos próprios professores da disciplina "Enfermagem em Saúde Mental”, por meio de cursos sobre as questões relacionadas à saúde mental, as habilidades que podem ser desempenhadas, o envolvimento com grupos de apoio e a busca por novas oportunida- des que possam ajudar no desenvolvimento e aprimoramento dos serviços voltados para a comunidade universitária ${ }^{(18)}$.

Na segunda categoria, "Outras demandas de Promoção da Saúde”, encontram-se artigos relacionados à demanda dos estudantes por outros serviços e necessidades de saúde, como atendimentos ambulatoriais, feiras de saúde e encontros reunindo os universitários para abordagem de algumas temáticas.

Uma universidade levantou um diagnóstico da sua população de estudantes, identificando que $70 \%$ do grupo de estudantes analisados apresentava algum distúrbio de saúde. O modelo de Promoção da Saúde preconizado incorpora a Enfermagem e Treinamento Esportivo, o que fortalece a autoestima e consciência de um estilo de vida saudável aos acadêmicos ${ }^{(12)}$.

Outras intervenções realizadas tinham a finalidade de reduzir o consumo de álcool, tabaco e maconha entres os estudantes ${ }^{(17)}$. Foi realizada uma palestra intitulada "Consumo de álcool e suas consequências", entrega do folder e cartilha educativa. Um ano após, realizaram a $2^{\mathrm{a}} \mathrm{IB}$, com novo feedback do resultado do padrão de consumo excessivo que cada universitário apresentou, sendo reorientados para leitura da cartilha educativa $^{(17)}$.

Outra promoção de saúde pela universidade integrou a saúde física dos graduandos de enfermagem com o serviço de saúde comportamental, estudo realizado em três universidades. Essa investigação aconteceu devido aos problemas encontrados entre os graduandos, tais como, dificuldade de aprendizagem, transtorno de déficit de atenção e hiperatividade, abuso de álcool e outras drogas ${ }^{(19)}$.

Devido a tais problemas, estas instituiçôes desenvolveram serviços de atendimento ambulatorial e diversas atividades de saúde mental (encontros com o grupo de universitários) com os discentes. A equipe responsável pelos atendimentos aos estudantes de enfermagem estava composta por psiquiatras, psicólogos, psicoterapeutas, enfermeiros e administrativos ${ }^{(19)}$.

Outro estudo relatou a respeito da prestação de serviços de saúde mental, a partir de encontros com os universitários em um Centro de Saúde e compreendeu-se que a presença dos enfermeiros nestes Centros promove comportamentos de saúde mais saudáveis ${ }^{(20)}$.

Em mais um estudo de 2007, encontrou-se mais uma ação de Promoção da Saúde voltada para assistência sexual de homens que fazem sexo com outros homens, profissionais do sexo, jovens e estudantes universitários, incluindo o curso de enfermagem. Tais ações realizadas consistiam em consultas, orientações em saúde, oferta de exames, preservativos e material educativo ${ }^{(21)}$.

A partir das atividades de promoção da saúde realizadas por professores de enfermagem, foram levantadas outras necessidades de serviços dentro da universidade para serem desenvolvidos com seus discentes, tais como: serviço de saúde mental, serviço de informações para o aprimoramento do bem estar dos discentes, serviços de atenção primária em saúde para manutenção e melhoria da saúde destes e o aconselhamento ${ }^{(23)}$.

Ainda nesta categoria temática, é analisado um estudo sobre a elaboração de uma feira de saúde em toda a universidade. Antes da elaboração dessa atividade foram identificadas quais as maiores demandas de saúde presentes nesta comunidade universitária, incluindo os discentes de enfermagem. Após esse levantamento, a feira disponibilizou serviços de atenção primária em saúde, conscientização sobre álcool, nutrição, saúde cardiovascular, prevenção e transmissão de infecções, massagem terapêutica, terapias alternativas, planejamentos e prevenção de doenças sexualmente transmissíveis ${ }^{(25)}$.

Finalizando esta categoria, um dos estudos relatou a percepção das mulheres universitárias sobre a saúde ginecológica. As participantes relataram que o estresse, as limitações de tempo e condições da vida universitária têm influenciado negativamente a sua capacidade de viver de forma saudável ${ }^{(22)}$.

Nesse sentido, a instituição pesquisada dispôs uma Clínica de Saúde Feminina, que presta atendimento às discentes de enfermagem e dos demais cursos. Dentre as atividades desenvolvidas nas consultas 
nessa clínica especializada têm-se exames ginecológicos de rotina para a prevenção do câncer, cuidados de saúde em geral com informações para tomadas de decisóes ${ }^{(22)}$.

\section{DISCUSSÃO}

A universidade torna-se o ambiente no qual o graduando de enfermagem, durante determinado período da vida, passa grande parte do seu tempo, sendo dessa forma um local apropriado para fazer e formar para a saúde, conscientizando-o do seu próprio bem estar físico, mental e social. Entende-se que as múltiplas responsabilidades inerentes ao período universitário poderão influenciar a saúde e o estilo de vida dos graduandos, refletindo nos aspectos pessoais e profissionais do presente e do futuro.

"A experiência universitária favorece a vulnerabilidade do adulto jovem para condutas e ambientes de risco a saúde"(26). É importante reforçar a adoção de medidas de prevenção e promoção da saúde, que integram questões ambientais, culturais e socioeconômicas. Atividades como cursos, consultas, encontros, workshops e feiras apresentam-se como estratégias possíveis para se alcançar esse objetivo dentro das universidades.

As experiências dos programas de saúde nas universidades são numerosas em diferentes modalidades, desenvolvidas com as funções de contribuir para o bem-estar universitário por meio de atividades assistenciais e de promoção e prevenção, com diferentes níveis de aplicação e cobertura social. As demandas internas de saúde universitária são atendidas por meio dos serviços de medicina geral, saúde bucal, saúde mental, saúde da gestante, primeiros socorros, entre outros ${ }^{(27)}$.

$O$ reconhecimento das necessidades reais dos graduandos de enfermagem é fundamental para que se inicie o planejamento e desenvolvimento de atividades que poderão repercutir positivamente na vida de tais indivíduos. É necessário fazer este levantamento de informações antes de se iniciar qualquer ação, pois as diferenças socioeconômicas, ambientais e culturais poderão exercer influência nas demandas de cada instituição.

A atenção voltada para a Saúde da Mulher é considerada como uma das prioridades de pesquisa pelo governo brasileiro. Tal programa ganhou destaque sendo elaborada a Política Nacional de Atenção Integral à Saúde da Mulher. Nesta política há a incorporação de temas norteadores como gênero, integralidade e a promoção da saúde. Os primeiros programas que abordavam Saúde da Mulher tinham um enfoque voltado apenas para a saúde reprodutiva e atenção obstétrica. A partir dessa Política avançou-se no campo dos direitos sexuais, planejamento familiar, abortamento, combate a violência doméstica e sexual, doenças, meio ambiente, lazer, alimentação, dentre outros ${ }^{(28)}$.

\section{CONCLUSÃO}

A presente Revisão Integrativa atingiu seus objetivos uma vez que possibilitou analisar a produção científica das atividades de Promoção da Saúde oferecidas pelas Universidades aos discentes de enfermagem; e gerar evidências dessas intervenções, tendo em vista o movimento das Universidades Promotoras da Saúde.

A pesquisa realizou uma síntese do conhecimento proveniente da produção científica internacional sobre o tema, sendo possível compreender atividades de Promoção da Saúde ofertadas pelas Universidades aos discentes de enferma- gem, envolvendo o desenvolvimento de programas, cursos, consultas, workshops e encontros.

A pesquisa identificou aspectos relevantes na promoção da saúde de graduandos de enfermagem, valorizando questões comportamentais dentro e fora da universidade. Ficou evidente a necessidade de ampliar as discussões de cuidado e ações com os estudantes que apresentam transtornos mentais cada vez mais comuns na atualidade, como ansiedade e depressão. A sensação de nervosismo e preocupação com os semestres, a extensa carga horária acadêmica e as diversas responsabilidades e deveres dentro da universidade, podem ter um impacto negativo na saúde física e mental, mas pode ser atenuado com promoção de práticas que facilitam a interação social e relaxamento.

Esta pesquisa demonstrou a necessidade de realização de novos estudos sobre a temática, que contribuam para a construção de políticas e ações de saúde voltadas para a população universitária de enfermagem que necessita ser atendida em sua singularidade.

Verificou-se ainda que as estratégias de Promoção da Saúde oferecidas pelas universidades aos graduandos de enfermagem demonstram preocupação com aspectos pontuais do corpo do estudante, mas nem sempre resulta da articulação entre as demandas estudantis.

A Universidade é um local apropriado para identificação das necessidades de saúde dos graduandos de enfermagem que deveria fundamentar o desenvolvimento de atividades de promoção da saúde. Dessa forma, conclui-se que existe uma preocupação, ainda que remota, das Universidades quanto à realização de atividades que buscam impactar positivamente o desenvolvimento humano saudável, consciente e sustentável dos futuros enfermeiros.

\section{REFERÊNCIAS}

1.Alayo M, Bambarén E, Gheiler M, Magill F, Sueiro R, Valdivieso $M$, et al. Guia para universidades saudáveis. 1. ed. Lima: Ed. Consorcio de Universidades; 2013.
2.Czeresnia D, Freitas CM. Promoção da saúde: conceitos, reflexões e tendências. 2. ed. Rio de Janeiro: Editora Fio Cruz; 2016. 


\section{REFERÊNCIAS}

3.Arroyo HA, Landazabal GD, Pino CG. Dez anos do movimento de universidades promotoras de saúde na Iberoamerica e a contribuição da Rede Iberoamericana de universidades promotoras de saúde (RIUPS). Rev. Global Health Promotion. 2015; 22(4): 64-8.

4.Okanagan Charter. Na international charter hor health promoting universities e colleges [Internet]. In: International Conference on Health Promoting Universities and Colleges/ VII International Congress. Kelowna, British Columbia: Canada; 2015 [acesso em 10 abr 2020]. p. 12. Disponível em: https:// www.fundadeps.org/recursos/documentos/735/carta-okanagan.pdf.

5.Declaración de Alicante. Declaración de Alicante sobre laPromoción de la Salud y Universidad. In: VIII Congresso IberoAmericano Universidades Promotoras de la Salud [Internet]. Alicante: Espanã; 2017 [acesso em 10 abr 2020]. p. 3. Disponivel em: http://www.um.es/documents/4856678/5231793/ DeclaracionAlicante2017.pdf/598e0885-0ffa-48c8-a3da-3963d5c8798f.

6.Freire P. Pedagogia da autonomia: saberes necessários à prática educativa. 53. ed. São Paulo: Editora Paz e Terra; 2016.

7 World Health Organization-WHO. Healthy Settings [Internet]. Geneve: WHO; 2016 [acesso em 10 abr 2020]. Disponível em: http:// www.who.int/healthy_settings/en/.

8- World Health Organization-WHO. 9th Global conference on health promotion: Global leaders agree to promote health in order to achieve Sustainable Development Goals. Geneve: WHO; 2016.

9. Ferreira FMPB, Brito IS, Santos MR. Health promotion programs in higher education: integrative review of the literature. Rev Bras Enferm. 2018; 71(4):1714-23.

10.Polit D, Beck CT. Fundamentos de pesquisa em enfermagem. 7. ed. Porto Alegre: Editora Artmed; 2011.

11.Melnyk BM, Fineout- Overholt E, Stillwell SB, Williamson KM. The Seven Steps of Evidence-Based Practice: Following this progressive, sequential approach will lead to improved health care and patient outcomes. American Journal of Nursing (AJN). 2010; 110(1): 51-3.

12- Aparicio CXP, Hernández FJB, Tamayo YG. Modelo de promoción de salud en la Universidad Metropolitana de Ecuador. Medisur. 2019; 17(6): 903-6

13- Burgess AW, Thabault P, Kiernan L, Flaherty E, Leestma K. College Warrior Athlete Initiative and academic nursing. J Am Coll Health. 2019; 12: 1-8

14- Liang HF, Wu KM, Hung CC, Wang YH, Peng NH. Resiliebce enhancement among student nurses during clinical practices: A participatory action research study. Nurse Educ Today. 2019; 75: 22-7.

15- Wichmann F, Braun M, Ganz T, Lubasch J, Heidenreich T, Laging $\mathrm{M}$ et al. Assessment of campus community readiness for tailoring implementation of evidence-based online programs to prevent risky substance use among university students in Germany. TranslBehay Med. 2019; 10(1): 114-122.

16- McCabe B, Troy A, Patel H,Halstead V, Arana M. Development of a university campus healthy sleep promotion program. Journal Issues in Mental Health Nursing. 2018;39(3): 264-8.

17- Sawicki WC, Fram DS, Belasco AGS. Intervenção breve aplicada a universitários consumidores de bebidas alcoólicas. Rev. Eletrônica Saúde Mental Álcool Drog. 2018; 1494): 22633.

18- Cook L. The role of psychiatric nurse faculty in establishing a campus suicide prevention program. J. Psychosoc Nurs.Ment Health Serv. 2011; 49(12): 22-8

19- Manderscheid RW, MasiD,Rossignol CR, Masi DA. The integration of physical health and behavioral health services: three university case examples. Arch PsychiatrNurs. 2007; 21(3): 141-9.

20 - Sturrock CJ, Currie MJ, Vally H, O'keefe EJ, Primrose R, Habel $P$ et al. Community-based sexual health care works: a review of the ACT outreach program. Sex Health. 2007; 4(3): 201-4.

21- Canales MK, CarrJ,Wohlberg, J. "It's about time": college women's perceptions of gynecologic health. J. Am. Acad. Nurse Pract. 2005; 17(6): 225-33.

22-Geary DM, Nardi DA, Smith J, Kremer M. Community need for university-based mental health services: identifying the top 5. 2005; 43(11): 38-45.

23- Clemmens D, Engler A, Chinn PL. Learning and living health: college students ${ }^{\prime}$ experiences with an introductory health course. J. Nurs Educ. 2004; 43(7): 313-8.

24- Gosline MB, Schank MJ. A university-wide health fair: a health promotion clinical practicum. Nurse Educ. 2003; 28(1): 23-5.

25.Moniz MA, Sabóia VM, Daher DV, Pereira RL, organizadores. Práticas Comunicativas socioambientais: inovação e potencialidades no uso de tecnologias educacionais na formação do enfermeiro. Niterói: Eduff; 2017.

26.Ministerio de Salud (PE), Dirección General de Promoción de la Salud. Promoviendo universidades saludables. Lima; 2010.

27.Ministério da Saúde (BR), Secretaria de Atenção à Saúde, Departamento de Ações Programáticas e Estratégicas. Política Nacional de Atenção Integral à Saúde da Mulher: princípios e diretrizes. Brasília (DF); 2004.

28. Oliveira CS. A Universidade Promotora de Saúde: uma revisão de literature [dissertação]. Salvador (BA): Instituto de Humanidades, Artes e Ciências, Universidade Federal da Bahia; 2017. 